\title{
Respiratory function in patients with thalassaemia and iron overload
}

\author{
I. Dimopoulou*, D.T. Kremastinos*, T.G. Maris*, S. Mavrogeni*, G.E. Tzelepis**
}

\begin{abstract}
Respiratory function in patients with thalassaemia and iron overload. I. Dimopoulou, D.T. Kremastinos, T.G. Maris, S. Mavrogeni, G.E. Tzelepis. CERS Journals Ltd 1999.

ABSTRACT: Iron deposition in the respiratory system has been proposed as a potential cause of the ventilatory restrictive impairment seen in patients with thalassaemia major (TM) and iron overload. In this study, magnetic resonance imaging (MRI) measurements of the liver (T2 relaxation time) were used as a surrogate index of total body iron burden and the extent to which these measurements correlated with total lung capacity (TLC) in patients with TM was examined.

Twenty-one patients (aged $25 \pm 5$ yrs) with TM participated in the study. Standard pulmonary function tests were undertaken and the $\mathrm{T} 2$ relaxation time of the liver was measured in all patients.

Ventilatory restrictive impairment (mean TLC $74 \pm 11$ (SD)\% predicted) was the most common abnormality found in $71 \%$ of $T M$ patients. There was no correlation between TLC (\% pred) and $\mathrm{T} 2$ relaxation time $(\mathrm{r}=0.06, \mathrm{p}=0.78)$. $\mathrm{T} 2$ relaxation time correlated weakly with average serum ferritin levels $(r=-0.56, p=0.008)$.

In conclusion, the data do not support the notion that the restrictive impairment in patients with thalassaemia major and iron overload is related to iron deposition in the respiratory system.

Eur Respir J 1999; 13: 602-605.
\end{abstract}

*Onassis Cardiac Centre and Aretaion Hospital, and University of Athens Medical School, Athens, Greece. **Wayne State University, Detroit, MI, USA.

Correspondence: I. Dimopoulou

Evgenidio Hospital

20 Papadiamantopoulou Street

GR 11528 Athens

Greece

Fax: 3017242785

Keywords: Iron deposition

magnetic resonance imaging

pulmonary function

thalassaemia

Received: June 301998

Accepted after revision November 71998

Abnormalities of respiratory function occur frequently in patients with thalassaemia major (TM) and include restrictive and or obstructive ventilatory defects, as well as an impairment of transfer factor (diffusing capacity) for carbon monoxide $[1,2]$. Of these, restrictive abnormalities are the most frequent, being reported in up to $80 \%$ of patients [1].

The precise aetiology of the restrictive abnormality remains unknown. A recent study [1] showed an inverse correlation between total lung capacity (TLC) and lifetime estimates of transfusional iron load and suggested that iron deposition in the lungs may play a key role in the pathogenesis of volume restriction in these patients. However, in another study [2], the calculated transfusional iron load did not correlate with TLC.

In this study, the issue of respiratory dysfunction in TM and its possible association with iron overload was re-examined. Unlike previous studies, iron overload was assessed by using magnetic resonance imaging (MRI) measurements of hepatic iron deposition rather than calculating the lifetime transfusional iron.

\section{Methods}

\section{Patients}

Twenty-one patients with homozygous $\beta$-thalassaemia participated in the study. All were being managed according to a hypertransfusion protocol designed to maintain a haemoglobin $(\mathrm{Hb})$ concentration $\geq 10 \mathrm{~g} \cdot \mathrm{dL}^{-1}$. Each patient was receiving chelation therapy with subcutaneous administration of desferrioxamine 5-7 times weekly. Fifteen patients had previously undergone splenectomy and seven patients had congestive heart failure (CHF), as assessed by clinical criteria and echocardiographic evidence of an ejection fraction of $<40 \%$. At the time of the study, all patients were clinically stable, with a mean $\mathrm{Hb}$ of $10 \pm 1$ $\mathrm{g} \cdot \mathrm{dL}^{-1}$. The patients with CHF were receiving conventional therapy for heart failure including digitalis, angiotensinconverting enzyme inhibitors and diuretics. The study protocol was approved by the Institutional Review Board.

\section{Pulmonary function and respiratory muscle strength tests}

Pulmonary function testing included measurement of spirometry, lung volumes and single-breath transfer factor for carbon monoxide (TL,CO) using a Jaeger system (Masterlab; Jaeger, Würzburg, Germany). TLC was measured by helium dilution and TL,CO was corrected for anaemia according to Cotes [3]. Transfer coefficient $(K \mathrm{CO})$ was calculated by dividing TL,CO by the alveolar volume. In each patient, the lower limits of normal values were calculated from published reference equations [3] as the mean predicted minus $1.65 \mathrm{SD}$. For the purpose of this study, restrictive disease was defined as a reduction in TLC, diffusion impairment as a reduction in $K \mathrm{CO}$ and obstructive airway disease as reduced forced expiratory volume in one second/forced vital capacity ratio (FEV1/ FVC ratio). Respiratory muscle strength was assessed by measuring maximum static inspiratory pressure $(P \mathrm{I}, \max )$ 
and expiratory pressure $(P E, \max )$ at residual volume and TLC, respectively. Values of $P$ I,max and $P$ E, max were compared with those of a control group ( $\mathrm{n}=15$, five males) matched for age and height.

\section{Magnetic resonance imaging studies}

MRI studies were performed in all patients using a 0.5T superconducting imaging system (MR-Max-Plus, GE/ CGR). A body coil was used for both excitation and signal detection. A quadrature detection technique was also applied. First proton density-weighted (PD) and T2-weighted (T2w) axial images of the upper abdomen were obtained using a multislice-double-echo spin echo (SE) sequence with a repetition time $(t \mathrm{R})$ of $2,500 \mathrm{~ms}$ and two echo times ( $t \mathrm{E} 1$ and $t \mathrm{E} 2$ ) of 12 and $80 \mathrm{~ms}$, respectively. Seven consecutive axial slices with 7-mm slice thickness were used. Then the fourth (median) of the consecutive slices was selected and a single-slice-multiecho SE sequence was applied using $t \mathrm{R}$ of $2500 \mathrm{~ms}$ and 20 symmetrically repeatable echo times, each being a multiple of $12 \mathrm{~ms}$. These parameters were chosen on the basis of the authors' prior phantom studies, in which a mean accuracy of 5.5\% and a mean reproducibility of $4.5 \%$ were demonstrated for a range of T2 values (10-80 ms). For both sequences, the field of view was rectangular $(42 \mathrm{~cm})$ and the image reconstruction axis was $224 \times 160$. The longer anatomical axis (horizontal) was chosen as the frequency encoding axis and the shorter as the phase encoding axis. Respiratory phase encoding and presaturation pulses were used to compensate for respiratory motion and blood flow artefacts, respectively.

\section{Quantitative image analysis}

Hepatic T2 relaxation time was measured from the T2calculated imaging maps. These imaging maps were calculated post hoc using 10 consecutive $\mathrm{T} 2 \mathrm{w}$ images obtained by the single-slice-multiecho imaging technique. The T2w MRI images were transferred to a PC workstation using a calibrated high-resolution video frame capture device. Images were then archived as conventional imaging files (256 grey levels, tagged image format (TIF) file).

Assuming single exponential behaviour of all tissues, pixel signal intensity (SI) decays exponentially with echo time in the base images of a multiecho sequence [4]. This behaviour is shown by the superimposed graphs (fig. 1) for a chosen region of interest located at the periphery of the liver. The rate of exponential decay can thus be calculated by means of a mathematical fit on SI and $t \mathrm{E} 1$ data values (fig. 1). This parameter, known as T2 relaxation rate (R2, inverse of relaxation time T2) was evaluated using a nonlinear regression analysis-fitting algorithm [4]. Differences in R2 or T2 can be depicted in the late base images of a multiecho sequence by differences in grey scale; the darker the liver image the greater the iron content (shorter T2 liver value and steeper slope of decaying curve).

To measure hepatic T2 values, five regions of interest located at the periphery of the liver were selected and a mean T2 value calculated. These calculations were carried out by one of the authors (T.G. Maris) who was unaware of the patients' lung function and serum ferritin levels. In each
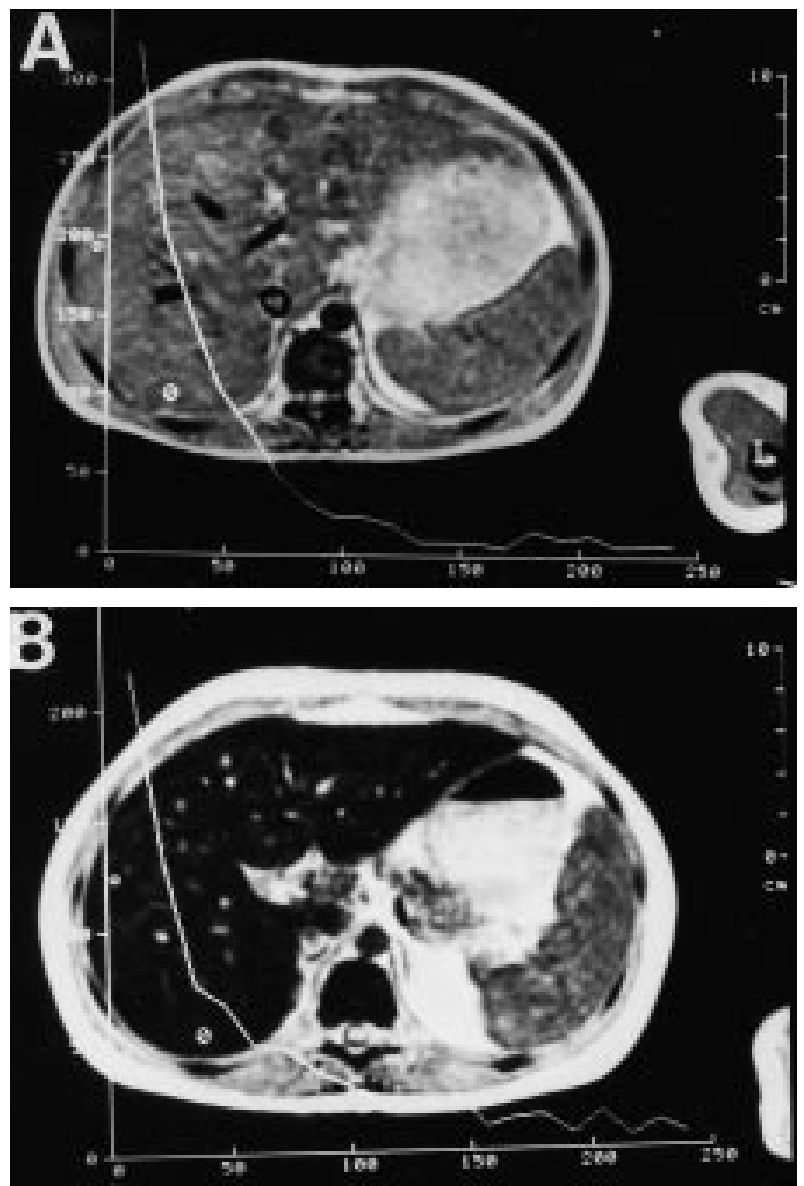

Fig. 1. - Representative T2-weighted magnetic resonance images of the liver in two patients with a) mild and b) heavy iron deposition. Note the darker image and steeper decay curve (i.e. shorter T2 value) in (b) (see text for more details).

patient, an average serum ferritin level was derived from approximately 30 values obtained bimonthly over the previous $5 \mathrm{yrs}$ and expressed in $\mathrm{ng} \cdot \mathrm{mL}^{-1}$ (normal values 20-200 ng.mL ${ }^{-1}$ ).

\section{Statistical analysis}

Data are presented as mean $\pm \mathrm{SD}$. Grouped data were compared using parametric or nonparametric tests, where appropriate. The Pearson product moment correlation coefficient was used to examine association between paired variables. All analyses were performed using a commercial software package (Sigmastat, Jandel, San Rafael, CA, USA). Significance was accepted at a p-value $<0.05$.

\section{Results}

Anthropometric and pulmonary function data are shown in table 1. Representative MRI in patients with mild or heavy iron overload are shown in figure 1 . The mean TLC for the entire TM patient group was mildly reduced ( $74 \pm 11, \%$ pred). On further analysis, only three of the 21 patients had normal lung function, with the remaining 18 patients $(85 \%)$ showing various isolated or combined functional abnormalities, with restriction and diffusion 
Table 1. - Anthropometric and respiratory function data

\begin{tabular}{|c|c|}
\hline Age yrs & $25 \pm 5$ \\
\hline Sex $M / F$ & $8 / 13$ \\
\hline Weight $\mathrm{kg}$ & $58 \pm 6$ \\
\hline Height $\mathrm{cm}$ & $164 \pm 12$ \\
\hline Smokers $\mathrm{n}$ & 9 \\
\hline FVC \% pred & $84 \pm 14$ \\
\hline FEV $1 \%$ pred & $82 \pm 15$ \\
\hline FEV1/FVC & $80 \pm 7$ \\
\hline FEF $25 \%-75 \% \%$ pred & $65 \pm 20$ \\
\hline TLC \% pred & $74 \pm 11$ \\
\hline$T \mathrm{~L}, \mathrm{CO} \%$ pred & $89 \pm 20$ \\
\hline$K \mathrm{CO} \%$ pred & $97 \pm 18$ \\
\hline$P \mathrm{I}, \max \mathrm{cmH}_{2} \mathrm{O}$ & $90 \pm 28$ \\
\hline$P \mathrm{E}, \max \mathrm{cmH}_{2} \mathrm{O}$ & $89 \pm 27$ \\
\hline
\end{tabular}

Data presented as mean \pm SD. MRI: Magnetic resonance imaging; $P$ I,max: maximum static inspiratory pressure; $P \mathrm{E}$,max: maximum static expiratory pressure; M: male; F: female; FVC: forced vital capacity; FEV1: forced expiratory volume in one second; FEF $25 \%-75 \%$ : forced mid-expiratory flow; TLC: total lung capacity; $T \mathrm{~L}, \mathrm{CO}$ : single-breath transfer factor for carbon monoxide; KCO: transfer coefficient.

impairment being the most frequent (table 2). In comparison with the control group, $P \mathrm{I}$,max and $P \mathrm{E}$,max were mildly reduced $\left(90 \pm 28\right.$ versus $108 \pm 19 \mathrm{cmH}_{2} \mathrm{O}$ for $P \mathrm{I}$,max and $89 \pm$ 27 versus $106 \pm 20 \mathrm{cmH}_{2} \mathrm{O}$ for $P \mathrm{E}, \max , \mathrm{p}<0.05$ for both).

Average serum ferritin was $2,669 \pm 1,237 \mathrm{ng} \cdot \mathrm{dL}^{-1}$ and hepatic T2 relaxation time was $30 \pm 11 \mathrm{~ms}$. There was a negative correlation between $\mathrm{T} 2$ relaxation time and serum ferritin $(\mathrm{r}=-0.56, \mathrm{p}=0.008)$. There was no correlation between TLC $(\%$ pred $)$ and age $(r=-0.17, p=0.45)$ or TLC $(\%$ pred) and serum ferritin $(\mathrm{r}=-0.17, \mathrm{p}=0.47)$. Hepatic T2 relaxation time did not correlate with TLC $\%$ pred $(\mathrm{r}=0.06$, $\mathrm{p}=0.78$, fig. 2$), T \mathrm{~L}, \mathrm{CO} \%$ pred $(\mathrm{r}=-0.06, \mathrm{p}=0.78)$ or with $K \mathrm{CO} \%$ pred $(\mathrm{r}=0.15, \mathrm{p}=0.53)$.

Comparison of respiratory function between patients with normal cardiac function $(n=14)$ with those with cardiac failure $(\mathrm{n}=7)$ showed no differences in TLC $(74 \pm 11$ versus $73 \pm 13 \%$ pred, $\mathrm{p}=0.82)$, FEV1 $(83 \pm 13$ versus $81 \pm$ $18 \%$ pred, $\mathrm{p}=0.73), \mathrm{FEV} 1 / \mathrm{FVC}(78 \pm 7$ versus $83 \pm 4 \%$ pred, $\mathrm{p}=0.12)$, KCO $(101 \pm 19$ versus $91 \pm 17 \%$ pred, $\mathrm{p}=0.27)$, TL,CO (82 \pm 28 versus $75 \pm 8 \%$ pred, $\mathrm{p}=0.48), P \mathrm{I}$,max $(92 \pm 30$ versus $\left.88 \pm 25 \mathrm{cmH}_{2} \mathrm{O}, \mathrm{p}=0.77\right)$ or $P \mathrm{E}, \max (94 \pm 27$ versus $\left.76 \pm 19 \mathrm{cmH}_{2} \mathrm{O}, \mathrm{p}=0.11\right)$. In patients with normal cardiac function, hepatic $\mathrm{T} 2$ relaxation time did not correlate with TLC $\%$ pred $(\mathrm{r}=0.13)$.

\section{Discussion}

The most prominent ventilatory defect affecting $71 \%$ of patients was restriction; about $24 \%$ of TM patients also showed a decrease in $\mathrm{KCO}$, suggesting parenchymal lung disease. None of these abnormalities was correlated with hepatic $\mathrm{T} 2$ relaxation time, an index of body iron overload.

Table 2. - Frequency of pulmonary function abnormalities in patients with thalassaemia major $(n=21)$

\begin{tabular}{lrr}
\hline Abnormality & $\mathrm{n}$ & $\%$ \\
\hline Restriction & 15 & 71 \\
Diffusion impairment & 5 & 24 \\
Obstruction & 2 & 9 \\
\hline
\end{tabular}

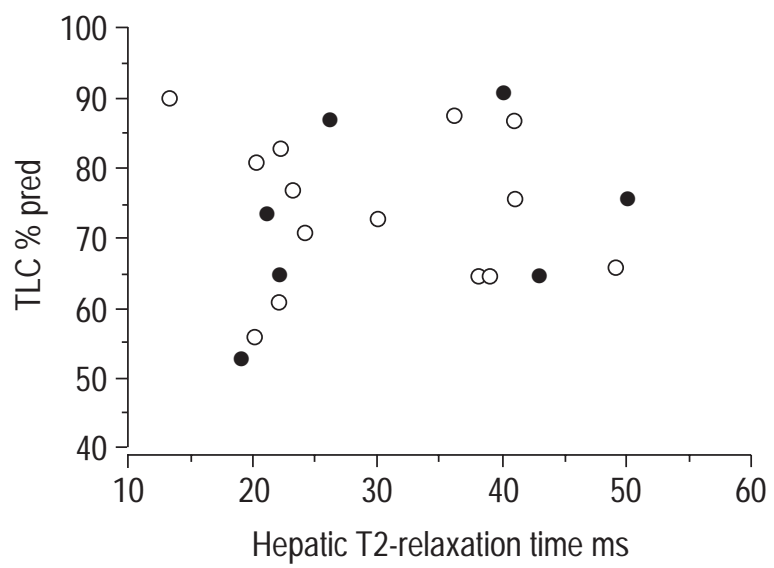

Fig. 2. - Relationship between total lung capacity (TLC) (\% predicted) and hepatic T2 relaxation time in thalassaemia patients with normal cardiac function $(\bigcirc)$ or with cardiac failure $(\bullet)$

Iron accumulation from repeated transfusions has been proposed as a likely cause of the restrictive defect in TM. Indeed, FACTOR et al. [1] reported an inverse relationship between TLC and estimates of transfusional iron burden (corrected for chelation therapy) and suggested that the degree and duration of iron overload might be important in the pathogenesis of the restrictive ventilatory defect. However, in a subsequent study by TAI et al. [2] the calculated lifetime iron burden did not correlate with the restrictive impairment in their patients. An inherent limitation of these calculations is that they do not take into account the amount of iron absorbed through the intestine, which in TM may be markedly increased and may itself lead to iron overload [5]. Furthermore, a cause-and-effect relationship of pulmonary iron deposition and restrictive abnormalities in TM is not supported by necropsy data reported in patients with siderosis. In these studies, iron was found predominantly in bronchial epithelial cells and bronchial glands rather than in the lung parenchyma $[6,7]$.

In the present study, body iron overload was assessed by means of MRI measurements of the hepatic T2 relaxation time. In general, measurement of the hepatic iron content gives the best quantitative estimate of body iron stores in patients with transfusional siderosis [8]. The MRI measurements are based on the ability of stored intracellular iron (ferritin and haemosiderin) to become strongly magnetized in a magnetic field. This magnetization is quantified by the magnetic susceptibility (the ratio of induced over the applied magnetic field). Localized regions of increased magnetic susceptibility selectively shorten $\mathrm{T} 2$ relaxation time by creating regions of magnetic field nonuniformity [9]. Recently, KALTWASSER et al. [10] reported a strong correlation between $\mathrm{T} 2$ relaxation time and hepatic iron concentration measured in biopsy specimens and a moderate correlation with serum ferritin. Likewise, GOMORI et al. [11] found a significant correlation between hepatic T2 relaxation time and iron concentration measured in surgical wedge samples from the liver. A prior study [4] found that liver T2 values correlated with hepatic iron concentration and with serum ferritin. These and other studies [12] have established that MRI is a reliable and useful noninvasive tool for quantifying hepatic iron in iron overload states.

Certain limitations of the present study should be acknowledged. Firstly, a fundamental assumption in this 
study is that iron deposition in the lung is proportional to that found in the liver. Given the inability to measure pulmonary iron concentration in vivo, the extent to which this assumption is valid may be open to criticism. However, necropsy data in TM patients with transfusional siderosis show that the iron content in the lung is generally a fixed proportion of that in the liver $[6,7]$.

The present $\mathrm{T} 2$ relaxation times represent mean values of five measurements obtained in the periphery of the liver. Since these measurements provide a global assessment of the hepatic iron deposition, they may have inaccurately assessed hepatic iron in cases with mild siderosis as well as in cases with heavy iron deposition in which the T2 values are usually very low and close to each other. However, further analysis of these two regions did not reveal any pattern of either normal or very abnormal TLC values. This again shows that in these patient subgroups TLC values are not tightly linked to iron deposition in the liver.

The present data confirm the findings of FACTOR et al. [1] that CHF does not appear to explain the restrictive defect in TM. In addition, they suggest that respiratory muscle strength is not greatly reduced to account for the diminished TLC. Although not specifically addressed in this study, the cause of the restrictive impairment requires further work. Previous studies have suggested other potential mechanisms including an underdevelopment of alveoli related to TM itself or to transfusion therapy $[1,13]$, and possibly toxicity related to desferrioxamine infusions [14].

In conclusion, using magnetic resonance imaging measurements of hepatic iron as a surrogate marker of total body iron content, no relationship between restriction of lung volume and measurements of hepatic iron was found in patients with TM. Thus, the data do not support the notion that the restrictive ventilatory impairment in thalassaemia is related to iron accumulation in the respiratory system.

\section{References}

1. Factor JM, Pottipati SM, Rappaport I, Rosner IK, Lesser ML, Giardina PJ. Pulmonary function abnormalities in thalassemia major and the role of iron overload. $\mathrm{Am} \mathrm{J}$ Respir Crit Care Med 1994; 149: 1570-1574.
2. Tai DYH, Wang YT, Lou J, Wang WY, Mak KH, Cheng HK. Lungs in thalassaemia major patients receiving regular transfusion. Eur Respir J 1996; 9: 1389-1394.

3. Cotes JE. Lung Function. Assessment and application in Medicine, 5th Edn. Oxford, Blackwell Scientific Publications, 1993.

4. Papakonstantinou OG, Maris TG, Kostaridou V, et al. Assessment of liver iron overload by T2-quantitative magnetic resonance imaging: correlation of T2-QMRI measurements with serum ferritin concentration and histologic grading of siderosis. Magn Reson Imaging 1995; 13: 967-977.

5. Pippard MJ, Callender ST, Warner GT, Weatherall DJ. Iron absorption and loading in beta-thalassaemia intermedia. Lancet 1979; 20: 819-821.

6. Cappell DF, Hutchison HE, Jowett M. Transfusional siderosis: the effects of excessive iron deposits on the tissues. $J$ Path Bact 1957; 74: 245-264.

7. Witzleben CL, Wyatt JP. The effect of long survival on the pathology of thalassaemia major. J Path Bact 1961; 82: 1-13.

8. Olivierin NF, Natham DG, MacMillan JH, et al. Survival in medically treated patients with homozygous $\beta$-thalassemia. $N$ Engl J Med 1994; 331: 574-578.

9. Johnston DL, Rice L, Vick GW, Hedrick TD, Rokey R. Assessment of tissue iron overload by nuclear magnetic resonance imaging. Am J Med 1989; 87: 40-47.

10. Kaltwasser JP, Gottschalk R, Schalk P, Hartl W. Noninvasive quantitation of liver iron-overload by magnetic resonance imaging. Br J Haematol 1990; 74: 360-363.

11. Gomori JM, Horev $\mathrm{G}$, Tamary $\mathrm{H}$, et al. Hepatic iron overload: quantitative MR imaging. Radiology 1991; 179: 367-369.

12. Ernst O, Sergent G, Bonvarlet P, Canva-Delcambre V, Paris JC, L'Hermine C. Hepatic iron overload: diagnosis and quantification with MR imaging. Am J Roentgenol 1997; 168: 1205-1208.

13. Cooper DM, Mansell AL, Weiner MR, et al. Low lung capacity and hypoxemia in children with thalassemia major. Am Rev Respir Dis 1980; 121: 639-646.

14. Freedman MH, Grisaru D, Olivieri N, MacLusky I, Thorner PS. Pulmonary syndrome in patients with thalassemia major receiving intravenous desferoxamine infusions. AJDC 1990; 144: 565-569. 